doi: $10.35366 / 94038$

https://dx.doi.org/10.35366/94038

ReVISIÓN - OPINIÓN

\title{
Infecciones asociadas al uso de fármacos anti-TNF en enfermedades reumáticas
}

\section{Infections associated with the use of anti-TNF drugs in rheumatic diseases}

\author{
Gabriel Cruz-López, * Diana Lucero Muñoz-Montes de Oca, * \\ Axel Torres-Monarrez, * Juan José Rubio-Castro, ${ }^{*}$ Elsa Janneth Anaya-Ambriz, ${ }^{*}$ \\ Gustavo Echeverría-González, ${ }^{\S}$ Juan Manuel Ponce-Guarneros, "César Arturo Nava-Valdivia"
}

\begin{abstract}
RESUMEN. El factor de necrosis tumoral alfa (TNF- $\alpha$ ) es una citocina proinflamatoria fundamental en la respuesta del sistema inmune contra las infecciones, además de participar en la patogénesis inflamatoria de enfermedades crónicas de origen autoinmune. Las terapias biológicas basadas en anticuerpos dirigidos a TNF- $\alpha$ son un valioso recurso en el tratamiento de enfermedades reumáticas. Sin embargo, esto conduce a un aumento en la susceptibilidad a infecciones ante patógenos oportunistas y complicaciones en estos pacientes. Esta revisión tiene como objetivo reportar evidencias acerca de las principales infecciones presentadas tras el uso de terapias anti-TNF- $\alpha$ en pacientes con enfermedades reumáticas, así como las estrategias realizadas ante estos casos.
\end{abstract}

Palabras clave: TNF- $\alpha$, infecciones, reumatología.

ABSTRACT. Tumor necrosis factor alpha (TNF- $\alpha)$ is a fundamental proinflammatory cytokine in the response of the immune system against infections, in addition to participating in the inflammatory pathogenesis of chronic diseases of autoimmune origin. Antibody-based biological therapies targeting TNF- $\alpha$ are a valuable resource in the treatment of rheumatic diseases. However, this leads to an increased susceptibility to infections with opportunistic pathogens and complications in these patients. The objective of this review is to report evidence on the main infections registered after the use of anti-TNF-a therapies in patients with rheumatic diseases, as well as the strategies carried out in these cases.

Keywords: TNF- $\alpha$, infections, rheumatology.

* Programa Nacional de Servicio Social en Investigación en Salud, Secretaría de Salud, México.

* Programa de Maestría en Microbiología Médica, Departamento de Microbiología, Centro Universitario de Ciencias de la Salud (CUCS), Universidad de Guadalajara, Guadalajara, Jalisco, México.

$\S$ Departamento de Reumatología, Unidad Médica de Alta Especialidad (UMAE), Centro Médico Nacional de Occidente (CMNO), Instituto Mexicano del Seguro Social (IMSS), Guadalajara, Jalisco, México.

" Departamento de Fisiología, Centro Universitario de Ciencias de la Salud (CUCS), Universidad de Guadalajara, Guadalajara, Jalisco, México.

Recibido: 29 de Enero de 2020.

Aceptado: 27 de Febrero de 2020.
『 Departamento de Microbiología, Centro Universitario de Ciencias de la Salud (CUCS), Universidad de Guadalajara, Guadalajara, Jalisco, México.

Correspondencia:

Dr. en C. César Arturo Nava-Valdivia

Calle Sierra Mojada No. 950, Edificio P, planta baja,

Independencia Oriente, CP. 44340, Guadalajara, Jalisco.

Tel: 01 (33) 10585309.

E-mail: dr.arturonaval@gmail.com

Conflicto de intereses:

Todos los autores declaran que no existe ningún conflicto de intereses con respecto a la publicación de este artículo. 


\section{INTRODUCCIÓN}

El factor de necrosis tumoral alfa (tumor necrosis factor $\alpha$ [TNF- $\alpha$ ], por sus siglas en inglés) es una citocina proinflamatoria fundamental en la respuesta del sistema inmune contra las infecciones. ${ }^{1} \mathrm{El}$ TNF- $\alpha$ incide en la actividad de otras citocinas inflamatorias como las interleucinas y quimiocinas (IL-1, IL-6 e IL-8), metaloproteinasas y óxido nítrico, importantes en el proceso de inflamación tanto aguda como crónica..$^{2,3}$

Además, participa en la patogénesis inflamatoria de enfermedades crónicas de origen autoinmune, entre las que destacan la artritis reumatoide, lupus eritematoso sistémico y espondilitis anquilosante. En estas enfermedades reumáticas el uso de terapias biológicas basadas en bloqueadores de TNF- $\alpha$ han demostrado gran eficacia para su tratamiento por disminución del proceso inflamatorio humoral, ya sea en monoterapia o en combinación con fármacos modificadores de la enfermedad (FARMES). Esto genera una mejoría clínica significativa, lo cual impacta en la calidad de vida de estos pacientes. ${ }^{4}$

En la actualidad, existen cinco medicamentos anti-TNF- $\alpha$ aprobados para su uso clínico en el campo de la reumatología: infliximab, adalimumab, certolizumab, golimumab y etanercept. Todos ellos anticuerpos monoclonales que inducen a una disminución de la actividad inflamatoria de TNF- $\alpha$ en sus formas transmembranales y solubles, que consecuentemente conllevan a apoptosis de linfocitos activados y disminución de la inflamación celular y humoral crónica en enfermedades reumáticas. ${ }^{5}$

Entre los efectos adversos más graves por el uso de estos fármacos son un aumento en la susceptibilidad a infecciones por patógenos oportunistas, principalmente intracelulares, tanto de etiología bacteriana como fúngica o vírica. ${ }^{6} \mathrm{Se}$ ha descrito que el uso de terapias anti-TNF- $\alpha$ incrementa el riesgo de infecciones hasta en $40 \%$, presentando variabilidad dependiente de la enfermedad reumática. ${ }^{7}$

Entre los principales microorganismos oportunistas reportados se encuentran bacterias como Mycobacterium tuberculosis, Legionella pneumophila y Listeria monocytogenes, hongos como Histoplasma capsulatum, Candida albicans y Pneumocystis jirovecii, y virus como herpes y hepatitis. La determinación de la tasa de infecciones en enfermedades reumáticas tratados con agentes biológicos anti-TNF- $\alpha$ resulta trascendental en el manejo de estos casos en la práctica clínica.

\section{INFECCIONES BACTERIANAS}

Las infecciones más comunes tras el uso de estas terapias son las de origen bacteriano, donde se ha observado que los órganos más afectados son el tracto respiratorio con una tasa de incidencia de 20.6, piel y tejidos blandos con 12.0 , hueso y articulaciones con 6.9 y tracto urinario con 4.6 , respectivamente. ${ }^{8}$

\section{Tuberculosis}

La tuberculosis (TB) es una infección adquirida mediante la exposición a Mycobacterium tuberculosis. Tras la inoculación a través del tracto respiratorio, la micobacteria es fagocitada por macrófagos alveolares, donde genera una inhibición de la acción degradativa del fagolisosoma, lo que favorece su multiplicación, generación de inflamación tisular, destrucción celular y formación de masas necróticas o granulomas, proceso mediado por la actividad proinflamatoria del TNF- $\alpha .{ }^{9}$

Esta citocina es esencial para la formación y estabilidad de granulomas, incrementando así el riesgo de infección o reactivación de tuberculosis latente. ${ }^{10,11}$

Se ha reportado que aproximadamente 90\% de los individuos infectados se encuentran en periodo de latencia sin presencia de sintomatología, por lo que en presencia de una enfermedad reumática tratada con fármacos anti-TNF se tiene un riesgo entre dos y cuatro veces mayor de progresión de infección respecto a los pacientes con otro tipo de tratamientos antirreumáticos. ${ }^{12}$ El riesgo relativo aumenta de 1.6 a 25 veces con el uso de terapia anti-TNF depen- 
diendo del contexto clínico, uso de drogas y procedencia del paciente. ${ }^{13}$

El riesgo de reactivación de TB latente depende a su vez del tipo de biológico empleado. Los anticuerpos monoclonales como el infliximab o adalimumab se han asociado con un aumento de cinco a 10 veces más riesgo de reactivación de tuberculosis; mientras etanercept se ha asociado a pocos casos de reactivación de tuberculosis. ${ }^{14}$

La administración de anti-TNF- $\alpha$ en casos positivos a TB debe ser postergada de 30 a 60 días. Si el paciente padece tuberculosis activa, la terapia biológica debe ser postergada por un periodo más largo hasta obtener la negatividad de los cultivos de esputo y mejoría clínica evidente. En este escenario el uso de otros agentes biológicos con dianas distintas como anakinra (anti-IL-1), tocilizumab (anti-IL-6), rituximab (anti-CD20), abatacept (anti-CD28), ustekinumab (anti-IL-12/23) o secukinumab (antiIL-17), deben ser priorizados para disminuir el riesgo de recaída. ${ }^{15,16}$

\section{Legionelosis}

Legionella pneumophila se ha identificado en múltiples ecosistemas, lagos, ríos o arroyos contaminados, spas, torres de enfriamiento, unidades de aire acondicionado y humidificadores. La infección intracelular en macrófagos alveolares conduce a neumonías necrosantes multifocales, por lo que es uno de los principales agentes etiológicos de neumonías intrahospitalarias y comunitarias. ${ }^{17}$

El uso de supresores de TNF- $\alpha$ ha demostrado una rápida diseminación y consecuentemente infecciones graves del tracto respiratorio, lo que aumenta la replicación de Legionella en el espacio intracelular de macrófagos y favorece la activación de apoptosis mediante caspasas. ${ }^{18}$

Se han reportado casos de neumonía causada por Legionella, registrados como eventos adversos tras el uso de infliximab. ${ }^{8}$ En 2011, la Food and Drug Administration (FDA) emitió un comunicado acerca del riesgo de infección por Legionella tras el uso de terapia anti-TNF- $\alpha$, el cual se recomienda ser postergado hasta la resolución de la infección. ${ }^{19}$

\section{Listeriosis}

Listeria monocytogenes es una de las principales bacterias asociadas con infecciones transmitidas por alimentos, debido a que se encuentra en el ambiente y en el tracto gastrointestinal de animales para consumo humano y sus derivados, como los lácteos no pasteurizados. Este microorganismo tiene la capacidad de ser fagocitado por macrófagos profesionales y no profesionales, y evadir su degradación intracelular, lo que favorece su multiplicación y posterior diseminación. ${ }^{20}$

Se ha demostrado que el uso de estas terapias disminuye de manera sustancial la resistencia a infecciones intracelulares por Listeria. ${ }^{21}$ Casos de infección han sido asociados con el tratamiento de fármacos como infliximab y etanercept en artritis reumatoide. ${ }^{21}$

Debido a la alta tasa de mortalidad que representa la infección por este microorganismo, el uso de bloqueadores de TNF- $\alpha$ resulta un factor de riesgo importante en aquellos pacientes reumáticos que reciben estos tratamientos. Infecciones por Listeria han demostrado una mortalidad hasta de $26 \%$ en pacientes bajo estos esquemas farmacológicos. ${ }^{22}$

Infecciones causadas por Listeria en pacientes reumáticos tratados con infliximab han reportado muertes por meningitis, endoftalmitis y sepsis, razón por la cual la FDA agregó a este patógeno las advertencias tras el uso de estos fármacos. ${ }^{23}$

\section{INFECCIONES MICÓTICAS}

\section{Histoplasmosis}

Histoplasma capsulatum es un hongo dimórfico, endémico de Norteamérica, Asia y parte de África, es el segundo microorganismo más frecuentemente aislado en pacientes con granulomatosis pulmonar que están bajo tratamiento con infliximab y etanercept, mediante un meca- 
nismo similar al que ocurre con Mycobacterium tuberculosis. ${ }^{14}$ Pacientes que residen en zonas endémicas desarrollan sintomatología entre la semana uno y 24 posteriores a la aplicación de la primera dosis. ${ }^{24}$

\section{Otras micosis oportunistas}

El uso de terapias biológicas en enfermedades reumáticas no han sido asociadas con infecciones localizadas o sistémicas causadas por Candida albicans. ${ }^{25}$

En cuanto a infecciones por Pneumocystis jirovecii, se han reportado neumonías relacionadas con el patógeno oportunista hasta en $86.5 \%$ de pacientes con artritis reumatoide bajo tratamiento con etanercept, y hasta $6.7 \%$ de mortalidad, debido a la severidad y rápido curso de la infección. ${ }^{26}$ La mayoría de estos eventos ocurrieron en pacientes que reciben otros agentes inmunosupresores como metotrexato o glucocorticoides. ${ }^{27}$

\section{INFECCIONES VIRALES}

\section{Herpes}

Los virus herpes provocan infecciones líticas en fibroblastos y células epiteliales, así como infecciones latentes en neuronas. Se ha propuesto que la participación del TNF- $\alpha$ es fundamental en la respuesta inflamatoria contra los virus herpes simple (VHS) tipo 1 y 2, así como en infecciones por virus herpes zóster (VHZ); por lo que la administración de fármacos anti-TNF- $\alpha$ se asocia con la exacerbación y recurrencia de la infección. ${ }^{28}$

Entre las principales manifestaciones clínicas reportadas tras el uso de infliximab en pacientes con artritis reumatoide se encuentran diseminación cutánea, herpes labial y encefalitis. ${ }^{29}$ Además, se han reportado casos aislados de encefalitis causada por VHS- 1 resistente a aciclovir tras el uso de adalimumab. ${ }^{30}$

En la actualidad, no está indicado el screening para VHS previo al inicio de terapia con anti-TNF- $\alpha$, por lo que la administración pro- filáctica de aciclovir deberá considerarse e individualizarse en pacientes con alto riesgo de recaídas. ${ }^{31}$

En el caso del VHZ, la reactivación de la infección latente es un problema de salud pública al ser causa de morbilidad, dolor crónico y discapacidad, debido a la neuralgia postherpética, particularmente en adultos mayores y pacientes inmunosuprimidos. ${ }^{32}$

Reportes indican que los pacientes con enfermedades reumáticas bajo tratamiento con antagonistas de TNF tienen mayor riesgo de hospitalización por reactivación de infecciones latentes graves por VHZ. ${ }^{33}$

En pacientes reumáticos existen varios factores que contribuyen a la reactivación de la enfermedad, entre los que destacan la edad avanzada, severidad de la enfermedad y dosis de glucocorticoides mayor de $10 \mathrm{mg} /$ día. ${ }^{34}$ Además, se ha demostrado que en artritis reumatoide y tratamiento anti-TNF representa un mayor riesgo de adquirir $\mathrm{VHZ}$ en comparación con tratamientos convencionales, esto debido a la inmunosupresión humoral del TNF- $\alpha \cdot{ }^{34-36} \mathrm{La}$ incidencia de infección no demostró diferencias entre el uso de etanercept en comparación con el uso de metotrexato en pacientes con artritis idiopática juvenil. ${ }^{37}$

Guías internacionales sugieren el considerar la vacunación contra VHZ en pacientes sin historia previa de la enfermedad cuatro semanas previas al inicio de la terapia biológica. ${ }^{31,38}$

\section{Hepatitis}

En contraste con lo descrito previamente, en la infección por virus de la hepatitis B (VHB), la inactivación del TNF- $\alpha$ podría incrementar la replicación viral y en consecuencia reactivar o complicar la infección. ${ }^{39}$ Sin embargo, las evidencias en pacientes con terapias anti-TNF e infección por VHB son escasos, contradictorios y no concluyentes respecto a la reactivación o exacerbación de la infección con el uso de estos tratamientos. ${ }^{40-42}$

En pacientes con infección por virus de la hepatitis $\mathrm{C}(\mathrm{VHC})$ crónica se han reportado niveles séricos elevados de TNF- $\alpha$, mismos que se 
han asociado en la patogénesis de la destrucción hepática y mayor letalidad tras la adquisición de infecciones. ${ }^{43}$ Además, niveles séricos altos de TNF- $\alpha$, previo al inicio de tratamiento antiviral con interferón (IFN) tienen menor probabilidad de respuesta al tratamiento contra VHC. ${ }^{44}$

En pacientes con artritis reumatoide con infección por VHC y tratados con anti-TNF- $\alpha$, se observó un aumento en niveles de linfocitos $\mathrm{T}$ CD4+ en sangre. ${ }^{45}$ Mientras que con el uso de etanercept, generó un aumento en la reactividad de linfocitos $\mathrm{T}$ a diversos antígenos microbianos en estos pacientes. ${ }^{46}$

Para otras infecciones virales, como virus de inmunodeficiencia humana (VIH) y VHC, es necesario realizar un tamizaje previo a la administración de agentes anti-TNF- $\alpha$ y tener en cuenta que la terapia antiviral deberá ser administrada previamente a la iniciación de esquemas con agentes anti-TNF, dando seguimiento al paciente con pruebas de función hepática y determinación de carga viral. ${ }^{47}$

\section{CONCLUSIÓN}

El uso de terapias biológicas anti-TNF- $\alpha$ en pacientes con enfermedades reumatológicas representa un factor de riesgo importante en la aparición de infecciones, esto debido a la inmunosupresión generada. En el caso de infecciones crónicas preexistentes en estos pacientes, se sugiere evaluar el uso y seguridad de estos tratamientos y, en medida de lo posible, postergar su uso hasta el control o resolución de la infección, así como dar seguimiento clínico, evaluar parámetros de laboratorio y monitorización de otros tratamientos anexos de manera individualizada. Además, es trascendental el mantener informado al paciente sobre factores de exposición a agentes patógenos y oportunistas para disminuir el riesgo de contagios.

\section{BIBLIOGRAFÍA}

1. Hehlgans T, Pfeffer K. The intriguing biology of the tumour necrosis factor/tumour necrosis factor receptor superfamily: players, rules and the games. Immunology. 2005; 115 (1): 1-20.

2. Taylor PC, Peters AM, Paleolog E, Chapman PT, Elliott MJ, McCloskey R et al. Reduction of chemokine levels and leucocyte traffic to joints by tumor necrosis factor alpha blockade in patients with rheumatoid arthritis. Arthritis Rheum. 2000; 43 (1): 38-47.

3. Ohshima S, Saeki Y, Mima T, Sasai M, Nishioka K, Ishida $\mathrm{H}$ et al. Long-term follow-up of the changes in circulating cytokines, soluble cytokine receptors and white blood cell subset counts in patients with rheumatoid arthritis (RA) after monoclonal anti-TNF-alpha antibody therapy. J Clin Immunol. 1999; 19 (5): 305-313.

4. Murdaca G, Spanò F, Contatore M, Guastalla A, Penza E, Magnani O, Puppo F. Infection risk associated with antiTNF- $\alpha$ agents: a review. Expert Opin Drug Saf. 2015; 14 (4): 571-582.

5. Thalayasingam N, Isaacs J. Anti-TNF therapy. Best Pract Res Clin Rheumatol. 2011; 25 (4): 549-567.

6. Winthrop KL. Risk and prevention of tuberculosis and other serious opportunistic infections associated with the inhibition of tumor necrosis factor. Nat Clin Pract Rheumatol. 2006; 2 (11): 602-610.

7. Rodrígez-Lozano C. Safety of biological therapies: new data from BIOBADASER. Reumatol Clin. 2011; 6S3: S1-6.
8. Dixon WG, Watson K, Lunt M, Hyrich KL, Silman AJ, Symmons DP. Rates of serious infection, including site-specific and bacterial intracellular infection, in rheumatoid arthritis patients receiving anti-tumor necrosis factor therapy: results from the British Society for Rheumatology Biologics Register. Arthritis Rheum. 2006; 54 (8): 2368-2376.

9. Tufariello JM, Chan J, Flynn JL. Latent tuberculosis: mechanisms of host and bacillus that contribute to persistent infection. Lancet Infect Dis. 2003; 3 (9): 578-590.

10. Roach DR, Bean AG, Demangel C, France MP, Briscoe H, Britton WJ. TNF regulates chemokine induction essential for cell recruitment, granuloma formation, and clearance of mycobacterial infection. J Immunol. 2002; 168 (9): 4620-4627.

11. Kapoor N, Pawar S, Sirakova TD, Deb C, Warren WL, Kolattukudy PE. Human granuloma in vitro model, for TB dormancy and resuscitation. PLoS ONE. 2013; 8 (1): e53657.

12. Doran MF, Crowson CS, Pond GR, O'Fallon WM, Gabriel SE. Frequency of infection in patients with rheumatoid arthritis compared with controls: a population-based study. Arthritis Rheum. 2002; 46 (9): 2287-2293.

13. Solovic I, Sester M, Gomez-Reino JJ, Rieder HL, Ehlers S, Milburn HJ, Kampmann B et al. The risk of tuberculosis related to tumour necrosis factor antagonist therapies: a TBNET consensus statement. Eur Respir J. 2010; 36 (5): 1185-1206. 
14. Wallis RS, Broder MS, Wong JY, Hanson ME, Beenhouwer DO. Granulomatous infectious diseases associated with tumor necrosis factor antagonists. Clin Infect Dis. 2004; 38 (9): 1261-1265.

15. Fernández-Ruiz M, Aguado JM. Risk of infection associated with anti-TNF-alpha therapy. Expert Rev Anti Infect Ther. 2018; 16 (12): 939-956.

16. Goletti D, Petrone L, Ippolito G, Niccoli L, Nannini C, Cantini F. Preventive therapy for tuberculosis in rheumatological patients undergoing therapy with biological drugs. Expert Rev Anti Infect Ther. 2018; 16 (6): 501-512.

17. Diederen BM. Legionella spp and Legionnaires disease. J Infect. 2008; 56 (1): 1-12.

18. Kawamoto Y, Morinaga Y, Kimura Y, Kaku N, Kosai $\mathrm{K}$, Uno N et al. TNF- $\alpha$ inhibits the growth of Legionella pneumophila in airway epithelial cells by inducing apoptosis. J Infect Chemother. 2017; 23 (1): 51-55.

19. Silver Spring, MD: US Food and Drug Administration. (2011). FDA drug safety communication: Drug labels for tumor necrosis factor-alpha $(\mathrm{TNF} \alpha)$ blockers now include warnings about infections with Legionella and Listeria bacteria.

20. Pillich H, Chakraborty T, Mraheil MA. Cell-autonomous responses in Listeria monocytogenes infection. Future Microbiol. 2015; 10 (4): 583-597.

21. Slifman NR, Gershon SK, Lee JH, Edwards ET, Braun MM. Listeria monocytogenes infection as a complication of treatment with tumor necrosis factor alpha-neutralizing agents. Arthritis Rheum. 2003; 48 (2): 319-324.

22. Schett G, Herak P, Graninger W, Smolen JS, Aringer M. Listeria-associated arthritis in a patient undergoing etanercept therapy: case report and review of the literature. J Clin Microbiol. 2005; 43: 2537-2541.

23. Kesteman T, Yombi JC, Gigi J, Durez P. Listeria infections associated with infliximab: case reports. Clin Rheumatol. 2007; 26 (12): 2173-2175.

24. Lee JH, Slifman NR, Gershon SK, Edwards ET, Schwieterman WD, Siegel JN et al. Life-threatening histoplasmosis complicating immunotherapy with tumor necrosis factor antagonists infliximab and etanercept. Arthritis Rheum 2002; 46: 2565-2570.

25. Aikawa NE, Rosa DT, Del Negro GM, Moraes JC, Ribeiro AC, Gonçalves SC et al. Systemic and localized infection by Candida species in patients with rheumatic diseases receiving anti-TNF therapy. Rev Bras Reumatol. 2016; 56 (6): 478-482.

26. Tanaka M, Sakai R, Koike R, Komano Y, Nanki T, Sakai $\mathrm{F}$ et al. Pneumocystis jirovecii pneumonia associated with etanercept treatment in patients with rheumatoid arthritis: a retrospective review of 15 cases and analysis of risk factors. Mod Rheumatol. 2012; 22 (6): 849-858.

27. Murdaca G, Negrini S, Pellecchio M, Greco M, Schiavi C, Giusti $\mathrm{F}$ et al. Update upon the infection risk in patients receiving TNF alpha inhibitors. Expert Opin Drug Saf. 2019; 18 (3): 219-229.

28. Van der Klooster JM, Bosman RJ, Oudemans-van Straaten HM, Van der Spoel JI, Wester JP, Zandstra
DF. Disseminated tuberculosis, pulmonary aspergillosis and cutaneous herpes simplex infection in a patient with infliximab and methotrexate. Intensive Care Med. 2003; 29 (12): 2327-2329.

29. Justice EA, Khan SY, Logan S, Jobanputra P. Disseminated cutaneous herpes simplex virus-1 in a woman with rheumatoid arthritis receiving infliximab: a case report. J Med Case Rep. 2008; 2: 282.

30. Schepers K, Hernandez A, Andrei G, Gillemot S, Fiten $\mathrm{P}$, Opdenakker G et al. Acyclovir-resistant herpes simplex encephalitis in a patient treated with antitumor necrosis factor- $\alpha$ monoclonal antibodies. J Clin Virol. 2014; 59 (1): 67-70.

31. Nordgaard-Lassen I, Frederik DJ, Belard E, Gerstoft J, Kjeldsen J, Kragballe K et al. Guidelines for screening, prophylaxis and critical information prior to initiating anti-TNF-alpha treatment. Dan Med J. 2012; 59 (7): 4480.

32. Winthrop KL, Baddley JW, Chen L, Liu L, Grijalva CG, Delzell E et al. Association between the initiation of antitumor necrosis factor therapy and the risk of herpes zoster. JAMA. 2013; 309 (9): 887-895.

33. García-Doval I, Pérez-Zafrilla B, Descalzo MA, Roselló R, Hernández MV, Gómez-Reino JJ et al. Incidence and risk of hospitalisation due to shingles and chickenpox in patients with rheumatic diseases treated with TNF antagonists. Ann Rheum Dis. 2010; 69 (10): 1751-1715.

34. Strangfeld A, Listing J, Herzer P, Liebhaber A, Rockwitz $\mathrm{K}$, Richter C. Risk of herpes zoster in patients with rheumatoid arthritis treated with anti-TNF- $\alpha$ agents. JAMA. 2009; 301 (7): 737-744.

35. Che H, Lukas C, Morel J, Combe B. Risk of herpes/ herpes zoster during anti-tumor necrosis factor therapy in patients with rheumatoid arthritis. Systematic review and meta-analysis. Joint Bone Spine. 2014; 81 (3): 215-212.

36. Liao TL, Chen YM, Liu HJ, Chen DY. Risk and severity of herpes zoster in patients with rheumatoid arthritis receiving different immunosuppressive medications: a case-control study in Asia. BMJ Open. 2017; 7 (1): e014032.

37. Nicolai R, Cortis E, Ravà L, Bracaglia C, Pardeo M, Insalaco A et al. Herpes virus infections during treatment with etanercept in juvenile idiopathic arthritis. Pediatric Infect Dis Soc. 2015; 5 (1): 76-79.

38. Rubin LG, Levin MJ, Ljungman P, Graham DE, Avery R, Tomblyn M et al. 2013 IDSA clinical practice guideline for vaccination of the immunocompromised host. Clin Infect Dis. 2014; 58 (3): 309-318.

39. Domm S, Cinatl J, Mrowietz U. The impact of treatment with tumour necrosis factor- $\alpha$ antagonists on the course of chronic viral infections: a review of the literature. Br J Dermatol. 2008; 159 (6): 1217-1228.

40. Michel M, Duvoux C, Hezode C, Cherqui D. Fulminant hepatitis after infliximab in a patient with hepatitis $B$ virus treated for an adult onset Still's disease. J Rheumatol. 2003; 30 (7): 1624-1625.

41. Oniankitan O, Duvoux C, Challine D, Mallat A, Chevalier $\mathrm{X}$, Pawlotsky JM et al. Infliximab therapy for rheumatic 
diseases in patients with chronic hepatitis B or C. J Rheumatol. 2004; 31 (1): 107-109.

42. Ostuni P, Botsios C, Punzi L, Sfriso P, Todesco S. Hepatitis B reactivation in a chronic hepatitis B surface antigen carrier with rheumatoid arthritis treated with infliximab and low dose methotrexate. Ann Rheum Dis. 2003; 62 (7): 6867.

43. Zylberberg H, Rimaniol AC, Pol S, Masson A, De Groote $\mathrm{D}$, Berthelot $\mathrm{P}$ et al. Soluble tumor necrosis factor receptors in chronic hepatitis C: a correlation with histological fibrosis and activity. J Hepatol. 1999; 30 (2): 185-191.

44. Fukuda R, Ishimura N, Ishihara S, Chowdhury A, Morlyama N, Nogami C et al. Intrahepatic expression of pro-inflammatory cytokine mRNAs and interferon efficacy in chronic hepatitis C. Liver. 1996; 16 (6): 390-399.
45. Maurice MM, Van der Graaff WL, Leow A, Breedveld FC, Van Lier RA, Verweij CL. Treatment with monoclonal anti-tumor necrosis factor alpha antibody results in an accumulation of Th 1 CD4+ T cells in the peripheral blood of patients with rheumatoid arthritis. Arthritis Rheum. 1999; 42 (10): 2166-2173.

46. Berg L, Lampa J, Rogberg S, Van Vollenhoven R, Klareskog $\mathrm{L}$. Increased peripheral $\mathrm{T}$ cell reactivity to microbial antigens and collagen type II in rheumatoid arthritis after treatment with soluble TNFalpha receptors. Ann Rheum Dis. 2001; 60 (2): 133-139.

47. Calabrese LH, Zein N, Vassilopoulos D. Safety of antitumour necrosis factor (anti-TNF) therapy in patients with chronic viral infections: hepatitis C, hepatitis B, and HIV infection. Ann Rheum Dis. 2004; 63 (2): 18-24. 